\title{
Artificial Intelligence in Health: New Opportunities, Challenges, and Practical Implications
}

\section{Findings from the Yearbook 2019 Section on Education and Consumer Health Informatics}

\author{
Annie Y. S. Lau', Pascal Staccini' ${ }^{2}$, Section Editors for the IMIA Yearbook Section on Education \\ and Consumer Health Informatics \\ 1 Centre for Health Informatics, Australian Institute of Health Innovation, Macquarie University, \\ Australia \\ 2 IRIS Department, URE RETINES, Faculté de Médecine, Université Côte d'Azur, France
}

Results: Only a small number of 2018 papers reported Artificial Intelligence (Al) research for patients and consumers. No studies were found on Al applications designed specifically for patients or consumers, nor were there studies that elicited patient and consumer input on Al. Currently, the most common use of Al for patients and consumers lies in secondary analysis of social media data (e.g., online discussion forums). In particular, the three best papers shared a common methodology of using data-driven algorithms (such as text mining, topic modelling, Latent Dirichlet allocation modelling), combined with insight-led approaches (e.g., visualisation, qualitative analysis and manual review), to uncover patient and consumer experiences of health and illness in online communities.

Conclusions: While discussion remains active on how Al could 'revolutionise' healthcare delivery, there is a lack of direction and evidence on how Al could actually benefit patients and consumers. Perhaps instead of primarily focusing on data and algorithms, researchers should engage with patients and consumers early in the Al research agenda to ensure we are indeed asking the right questions, and that important use cases and critical contexts are identified together with patients and consumers. Without a clear understanding on why patients and consumers need Al in the first place, or how Al could support individuals with their healthcare needs, it is difficult to imagine the kinds of Al applications that would have meaningful and sustainable impact on individual daily lives.

\section{Keywords}

Arrificial intelligence, consumer informatics, patient, participatory health, machine learning, social media, consumer

Yearb Med Inform 2019:174-80

http://dx.doi.org/10.1055/s-0039-1677935

\section{Introduction}

For this 28th edition of the Yearbook of Medical Informatics, the topic of "Artificial Intelligence (AI) in Health: New Opportunities, Challenges, and Practical Implications" is thought-provoking, especially when it comes to 're-imagining' the future for patients and consumers as AI technologies are increasingly introduced into our daily lives.

Over the past years, our ability to store large repositories of data has surpassed the ability to effectively and efficiently develop actionable knowledge from these sources.
For example, cloud artificial intelligence and machine learning platform services, known collectively as AI Platforms as a Service (PaaS), are placed at the peak of the Gartner hype curve in 2018 [1]. Due to the opportunity of having access to large quantities of data that were previously unavailable, and the technical challenge of not being able to deliver real-time actionable knowledge from these sources, many researchers have worked on enhancing machine learning algorithms to extract meaning from these sources. As a result, many of the AI applications we are witnessing today are reliant on having access to large repositories of data. Success of these data-driven approaches varies across disciplines and depends on the quality and quantity of the data available, the specificity of the task, the appropriate choice of algorithms, the rigour in the execution, as well as the domain expertise available to guide the analysis and interpretation.

In health, recent AI developments that are showing promising results are data-driven approaches, specifically for clinician-facing applications such as image analysis and interpretation in radiology $[2,3]$. In the world of patients and consumers, recent AI applications have also taken a data-driven approach. Social 
media platforms, including online health communities, have become popular sources for individuals to connect and exchange support. Due to the relative ease to access publicly-available data, many researchers have tapped into mining social media as a way to explore how AI can be applied to get a better understanding of patient and consumer experiences. As a result, a majority of recent studies reporting AI approaches for patients and consumers focuses on secondary analyses of social media data [4]. While social media could be a good source to understand how individuals cope with and manage their conditions, they also present high risks due to widespread dissemination of poor-quality or incorrect information. As a result, researchers have proposed various data-driven approaches to analyse patients' online behaviours and address the problems they experience online, such as detecting disclosure of personal health information on Twitter [5] and determining which online health forum threads require moderator assistance [6].

However, these data-driven approaches, regardless of whether they are focused on clinicians, consumers, or patients, represent a narrow focus of AI [7]. In this paper, we examine how AI approaches are currently used for patients and consumers, present papers that are representative in the year 2018, and highlight untapped opportunities for research in AI for patients and consumers.

\section{Methodology}

\subsection{Search Strategy}

We used PubMed to conduct our search, capturing papers relevant to consumer health informatics and artificial intelligence published in the year 2018. The search strategy was based on the PICO framework, P-Population/Problem, I-Intervention, C-Comparison, O-Outcome, where 'Problem' refers to the various digital environments consumers and patients participate in, e.g., social media, online health communities; "Intervention" comprises of various AI methods and technologies; and "Outcome" outlines the impact or resulting effects of participatory health, i.e., patient-centred initiatives to empower individ- uals in their health decisions and behaviours [4]. A "comparison" intervention was not included as it is not relevant in this review.

We started with a search query adopted from previous work $[2,4]$. Step by step, we refined the query to include keywords related to digital/social media (41 keywords), artificial intelligence (52 keywords), and participatory health (24 keywords). MeSH terms and the syntax "[All Fields]" were used wherever possible to ensure our search strategy was comprehensive. The final search query is listed below:

((2018[DP] NOT pubstatusaheadofprint) NOT Bibliography[pt] NOT Comment[pt] NOT Editorial[pt] NOT Letter[pt] NOT News[pt] NOT Case Reports[pt] NOT Published Erratum[pt] NOT Historical Article[pt] NOT legislation[pt] NOT ("review" [pt] OR "review literature as topic"[MeSH] OR "literature review"[All Fields]))

AND ("social media"[All Fields] OR "facebook"[All Fields] OR “twitter"[All Fields] OR "youtube"[All Fields] OR "instagram"[All Fields] OR "pinterest"[All Fields] OR "google trends"[All Fields] OR "snapchat"[All Fields] OR "whatsapp"[All Fields] OR "posts"[All Fields] OR "blog"[All Fields] OR "microblog"[All Fields] OR "wiki"[All Fields] OR "health communities"[All Fields] OR "social network site"[All Fields] OR "social web"[All Fields] OR "online social network"[All Fields] OR "social environment"[All Fields] OR "social process"[All Fields] OR "social competition"[All Fields] OR "social norm"[All Fields] OR "social feedback"[All Fields] OR "social influence"[All Fields] OR "social comparison"[All Fields] OR "social network"[All Fields] OR "discussion group"[All Fields] OR "support group"[All Fields] OR "social support"[All Fields] OR "community network"[All Fields] OR "online community"[All Fields] OR "second life"[All Fields] OR "virtual worlds"[All Fields] OR "virtual reality"[All Fields] OR "web 2.0"[All Fields] OR "web 3.0"[All Fields] OR "medicine 2.0"[All Fields] OR "health 2.0"[All Fields] OR "digital health"[All Fields] OR "platform"[All Fields] OR "nontraditional data sources"[All Fields] OR "novel data streams"[All Fields])

AND ("data science"[All Fields] OR "artificial intelligence"[All Fields] OR "learning systems"[All Fields] OR "big data"[All Fields] OR "machine learning"[All
Fields] OR "deep learning”[All Fields] OR "reinforcement learning"[All Fields] OR "supervised learning"[All Fields] OR "unsupervised learning"[All Fields] OR "active learning"[All Fields] OR "neural network"[All Fields] OR "convolutional neural network"[All Fields] OR "recurrent neural network"[All Fields] OR "natural language processing"[All Fields] OR "text mining"[All Fields] OR "support vector machine"[All Fields] OR "support vector network"[All Fields] OR "support vector classifier"[All Fields] OR "naive bayes"[All Fields] OR "bayesian network"[All Fields] OR "bayesian learning"[All Fields] OR "boosting”[All Fields] OR "machine intelligence"[All Fields] OR "computational intelligence"[All Fields] OR "decision tree"[All Fields] OR "ensemble trees"[All Fields] OR "random forest"[All Fields] OR "clustering"[All Fields] OR "classification"[All Fields] OR "validation"[All Fields] OR "first-order logic"[All Fields] OR "fuzzy model"[All Fields] OR "cellular automaton"[All Fields] OR "markov model"[All Fields] OR "swarm intelligence"[All Fields] OR "knowledge reasoning"[All Fields] OR "computational inference"[All Fields] OR "model stacking"[All Fields] OR "intelligent agent"[All Fields] OR "multiagent system" [All Fields] OR “conversational agent"[All Fields] OR "case-based reasoning"[All Fields] OR "rule-based system"[All Fields] OR "knowledge-based reasoning"[All Fields] OR "knowledge representation"[All Fields] OR "qualitative reasoning"[All Fields] OR "decision-theoretic planning"[All Fields] OR "computer reasoning"[All Fields] OR "prediction"[All Fields] OR "genetic algorithms"[All Fields] OR "evolutionary algorithms"[All Fields] OR "evolutionary computing"[All Fields])

AND ("digital behavior"[All Fields] OR "compliance"[All Fields] OR "observance"[All Fields] OR "pharmaco epidemiology"[All Fields] OR "digital epidemiology"[All Fields] OR "infoveillance"[All Fields] OR "participatory health"[All Fields] OR "participatory medicine" [All Fields] OR "patient engagement"[All Fields] OR "participatory medicine"[All Fields] OR "patient empowerment" [All Fields] OR "shared decision making" [All Fields] OR "patient-practitioner relationship" [All Fields] OR "consumer health" [All Fields] OR "consumer empow- 
Lau et al.

erment" [All Fields] OR "citizen health" [All Fields] OR "citizen engagement" [All Fields] OR "citizen empowerment" [All Fields] OR "participative medicine" [All Fields] OR "personalized medicine" [All Fields] OR "precision medicine" [All Fields] OR "predictive medicine" [All Fields] OR "preventive medicine" [All Fields] OR "disease management" [All Fields])

\subsection{Bibliometrics Analyses}

To understand the state of the literature, we applied various bibliometrics tools onto the original set of articles returned from the search query. The "Bibliometrix" package from R [8] was used on the retrieved articles to report frequency of keywords used by authors, and a topic dendrogram to cluster keywords and examine whether these clusters follow a hierarchical structure.

\section{Results}

\subsection{State of the Literature}

Ninety-nine articles were returned from the search query. Figure 1 reports the frequency of the 50 most common keywords used by authors in these retrieved articles. The five most frequent keywords are (from most frequent to least frequent): precision medicine, social media, big data, infodemiology, and machine learning, illustrating the current focus on data-driven approaches to AI for patients and consumers.
Figure 2 illustrates a dendrogram applied to the 99 retrieved articles to explore whether keywords used by authors tended to cluster in groups, and whether these clusters follow a hierarchical structure. Three small clusters emerged, illustrating the keywords that were commonly used together by authors when describing $\mathrm{AI}$ approaches for patients and consumers. For example: "machine learning" and "precision" in cluster 1; "infodemiology", "mortality", and "outcome" in cluster 2; and "forensic DNA phenotyping" and "DNA methylation" in cluster 3.

We did not observe a clear hierarchical structure amongst these clusters but "digital health" emerged as a unique term associated at the root of the tree. Furthermore, there is a list of keywords near the centre of the dendrogram that do not seem discriminant

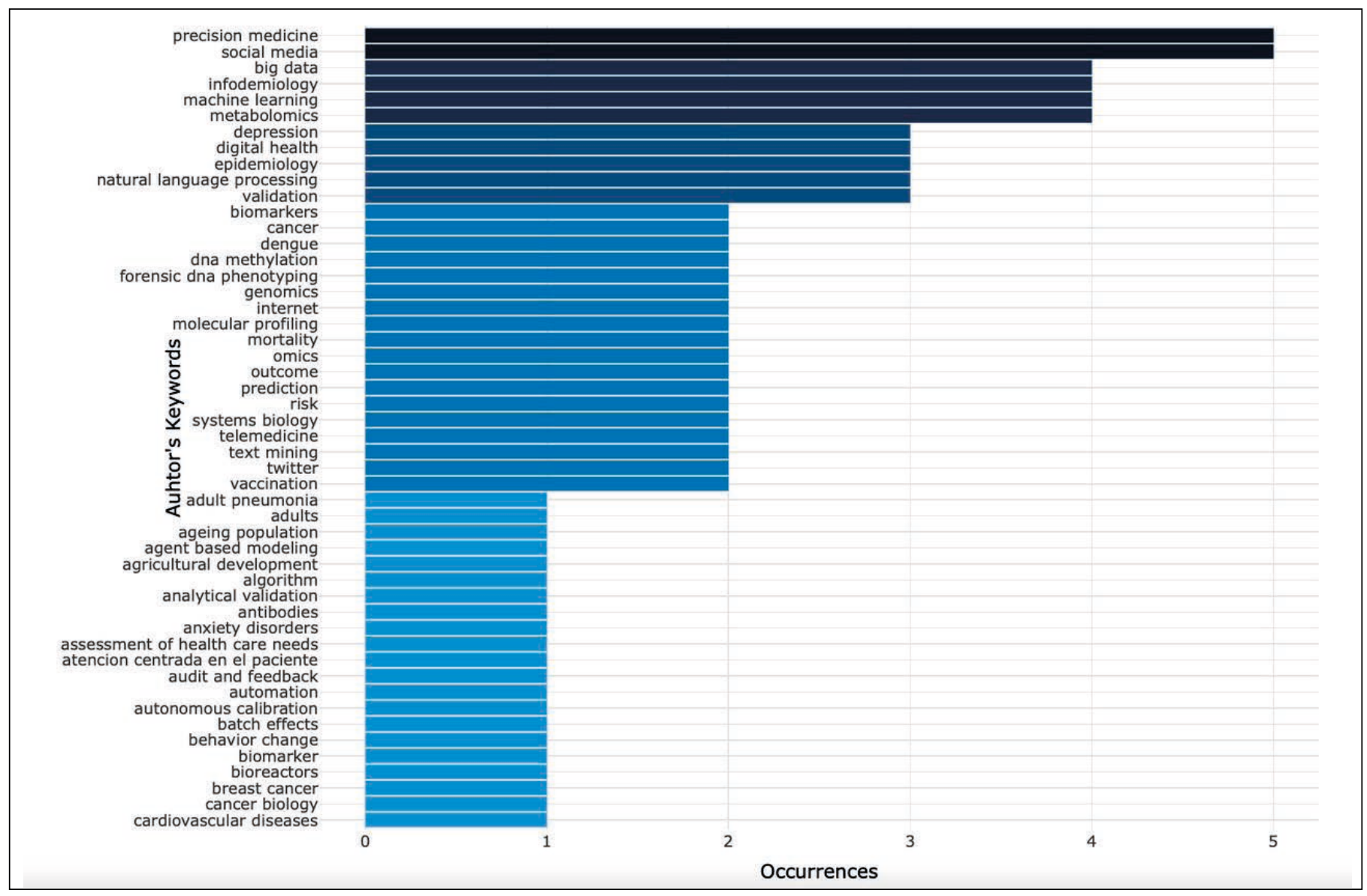




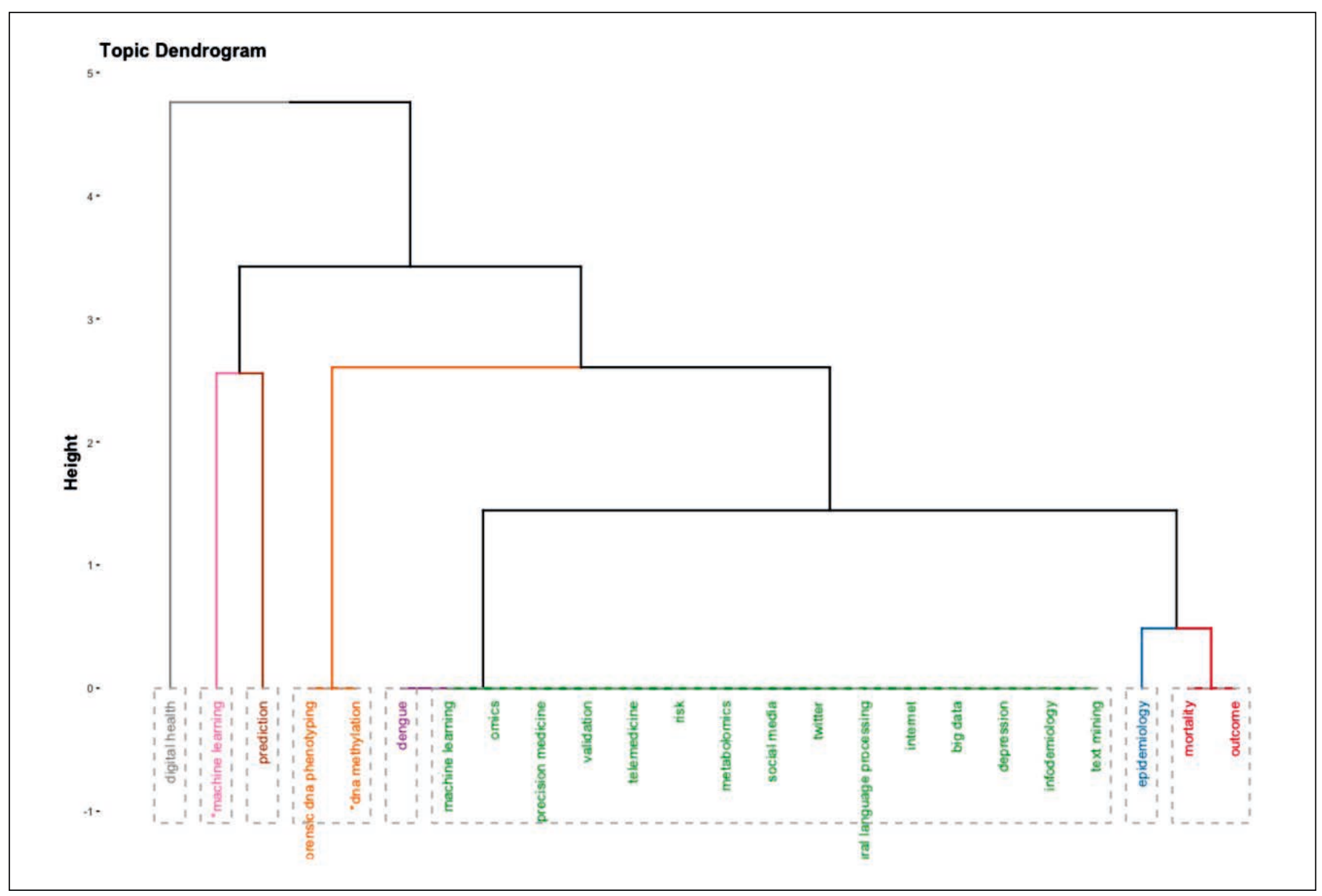

Fig. 2 Topic dendrogram of author's keywords throughout the 99 retrieved articles.

enough to form a cluster (e.g., natural language processing, big data, depression, twitter, internet, text mining, etc.). The inability to form clusters amongst these keywords may suggest that although there is research activity in each of these individual areas, different authors used different approaches and there is not yet a body of work that combines these concepts, methods, or techniques when authors describe their work in the literature.

\subsection{Best Paper Selection}

The 99 retrieved articles were then screened by section editors, which resulted in 14 articles considered for best paper selection. Elements that were considered in the screening decision include: 1) level of relevance regarding the 2019 yearbook topic "Artificial Intelligence in Health: New Opportunities, Challenges, and Practical Implications"; 2) whether the AI application was focused only on patients and consumers; 3 ) nature of the healthcare problem addressed; and 4) level of innovative approach.

The selected 14 articles were then presented to a panel of international experts for full paper review and scoring according to the IMIA Yearbook best paper selection process. The three papers that received the highest scores were then discussed in a consensus meeting, and it was agreed upon that they were representative papers on artificial intelligence in health for patients and consumers for the year 2018.

The final three best papers selected after peer review process are listed in Table 1.
These papers shared a common methodology of using data-driven algorithms (such as text mining, topic modelling, or Latent Dirichlet allocation modelling), combined with insight-led approaches (e.g. visualisation, qualitative analysis, or manual review), to uncover patient and consumer experiences of health and illnesses in online communities.

For example, Abdellaoui et al., [9] outlined a methodology to detect medication non-compliance behaviours amongst people on antidepressant and antipsychotic medications by modelling the way dosage variation and treatment interruption behaviours were discussed online. Jones et al., [10] demonstrated it was possible to uncover the hidden, less obvious aspects of breast cancer management and recovery in online discussion forums, including aspects that are not easily ascertainable 
Table 1 Best paper selection of articles for the IMIA Yearbook of Medical Informatics 2019 in the section 'Education and Consumer Health Informatics'. The articles are listed in alphabetical order of the first author's surname.

\section{Section \\ Education and Consumer Health Informatics}

- Abdellaoui R, Foulquié P, Texier N, Faviez C, Burgun A, Schück S. Detection of Cases of Noncompliance to Drug Treatment in Patient Forum Posts: Topic Model Approach. J Med Internet Res 2018;20(3):e85.

- Jones J, Pradhan M, Hosseini M, Kulanthaivel A, Hosseini M. Novel Approach to Cluster Patient-Generated Data Into Actionable Topics: Case Study of a Web-Based Breast Cancer Forum. JMIR Med Inform 2018;6(4):e45.

- Park A, Conway M, Chen AT. Examining Thematic Similarity, Difference, and Membership in Three Online Mental Health Communities from Reddit: A Text Mining and Visualization Approach. Comput Human Behav 2018 Jan;78:98-112.

in patient clinics (e.g., side effects while in remission, financial challenges experienced by cancer survivors over time). Similarly, Park et al., [11] identified subtle differences in the types of concerns expressed online by individuals experiencing different mental health conditions (e.g., people with depression often discussed events associated with changes in mood whereas discussion topics amongst people with anxiety or post-traumatic stress disorder clustered around treatment- and medication-related issues).

\section{Conclusions}

Despite the attention and expectations given to artificial intelligence (AI), we did not find eligible articles published in 2018 that reported AI applications designed specifically for patients or consumers, nor literature that elicited patient and consumer input on AI. Currently, the most common use of AI for patients and consumers lies in secondary analysis of social media data (e.g., online discussion forums). In particular, the three 2018 best papers share a common methodology of using data-driven algorithms, combined with insight-led approaches, to uncover patient and consumer experiences in online communities.

Currently, there is a lack of direction and evidence on how AI would actually benefit patients and consumers. Perhaps instead of focusing on data and algorithms, researchers should engage with patients and consumers early in the AI research agenda to ensure we are indeed asking the right questions, and that important use cases and critical contexts are identified together with patients and consumers. Without a clear understanding on why patients and consumers need AI in the first place, how AI could support individuals with their healthcare needs, and what are the capabilities and limitations of AI, it is difficult to imagine the kinds of AI applications that would have meaningful and sustainable impact on individuals' daily lives.

Artificial Intelligence in 2018 may not yet be at the state that meets the expectations of patients and consumers. However, this presents a number of untapped opportunities for research. While many have already made way in using data-driven and machine learning approaches in health, perhaps the challenge of AI for patients and consumers lies in how people will interact with the technology (i.e. human-computer interaction) [4]. For patients and consumers to truly benefit from $\mathrm{AI}$, the design of the technology may need to be embedded deeply in their environment or perhaps even invisibly in their daily routine [4]. For example, with the rise of voice-only or voice-first interfaces, one could explore whether conversational agents have a role to support patients and consumers with their daily tasks [12]. In addition, real-life decision support for patients and consumers remains an open opportunity provided the right problem, use case, and interaction mode are identified. To conclude, we leave readers with the words of Heht [13]: "The public's view of artificial intelligence might not be accurate, but that doesn't mean researchers can ignore it".

\section{References}

1. Sicular S, Brant K. Hype Cyle for Artificial Intelligence. Gartner, Inc; 2018. p. 24-5.

2. Lee Ventola C. Social media and health care professionals: Benefits, risks, and best practices. $P$ and T 2014;39(7):491-9.

3. Shaban-Nejad A, Michalowski M, Buckeridge DL. Health intelligence: how artificial intelligence transforms population and personalized health. Digital Medicine 2018;1(1):53.

4. Shaban-Nejad A, Lavigne M, Okhmatovskaia A, Buckeridge DL. PopHR: a knowledge-based platform to support integration, analysis, and visualization of population health data. Ann N Y Acad Sci 2017 Jan;1387(1):44-53.

5. Denecke K, Gabarron E, Grainger R, Konstantinidis ST, Lau A, Rivera-Romero O, et al. Artificial Intelligence for Participatory Health: Applications, Impact, and Future Implications. Yearb Med Inform 2019 Apr 25.

6. Yin Z, Fabbri D, Rosenbloom ST, Malin B. A Scalable Framework to Detect Personal Health Mentions on Twitter. J Med Internet Res 2015 Jun 5;17(6):e138.

7. Huh J, Yetisgen-Yildiz M, Pratt W. Text classification for assisting moderators in online health communities. J Biomed Inform 2013 Dec;46(6):998-1005.

8. Aria, M, Cuccurullo, C. Bibliometrix: An R-tool for comprehensive science mapping analysis. Journal of Informetrics 2017;11(4):959-75.

9. Abdellaoui R, Foulquié P, Texier N, Faviez C, Burgun A, Schück S. Detection of Cases of Noncompliance to Drug Treatment in Patient Forum Posts: Topic Model Approach. J Med Internet Res 2018;20(3):e85.

10. Jones J, Pradhan M, Hosseini M, Kulanthaivel A, Hosseini M. Novel Approach to Cluster Patient-Generated Data Into Actionable Topics: Case Study of a Web-Based Breast Cancer Forum. JMIR Med Inform 2018;6(4):e45.

11. Park A, Conway M, Chen AT. Examining Thematic Similarity, Difference, and Membership in Three Online Mental Health Communities from Reddit: A Text Mining and Visualization Approach. Comput Human Behav 2018 Jan;78:98-112.

12. Laranjo L, Dunn AG, Tong HL, Kocaballi AB, Chen J, Bashir R, et al. Conversational agents in healthcare: a systematic review. J Am Med Inform Assoc 2018 Sep 1;25(9):1248-58.

13. Hecht J. Meeting people's expectations. Nature 2018;563:S141-2.
Correspondence to:
Annie Y.S. Lau
Centre for Health Informatics
Australian Institute of Health Innovation
Macquarie University, Australia
E-mail:annielau@mq.edu.au 
Appendix: Content Summaries of Best Papers for the Education and Consumer Health Informatics Section of the 2019 IMIA Yearbook

\author{
Abdellaoui R, Foulquié $P$, Texier N, Faviez \\ C, Burgun A, Schück S \\ Detection of Cases of Noncompliance to \\ Drug Treatment in Patient Forum Posts: \\ Topic Model Approach
}

\section{J Med Internet Res 2018;20(3):e85}

Non-compliance (or non-adherence) to long-term treatment is a worldwide problem detrimental to the overall effectiveness of the health system. Social media holds a lot of promise in improving communication and patient engagement. The example of benfluorex illustrates how social media could be valuable sources for experts. Methods to identify messages with adverse events mentions have been developed and it has been showed that social media may even impact treatment adherence. The objective of this study was to evaluate a topic model approach to detect patient non-compliant behaviours (dose change and treatment cessation) associated with antidepressant drug (escitalopram) and antipsychotic drug (aripiprazole) in online forums. Authors implemented a probabilistic topic model to identify the topics that occurred in a corpus of messages mentioning these drugs, posted from 2004 to 2013 on three of the most popular French forums. Around 6\% (154/2691) of online posts were detected on escitalopram non-compliance and 7\% (122/1778) on aripiprazole. The topic models approach detected cases of non-compliance behaviours with average recall and precision scores of $98.5 \%$ (272/276) and $32.6 \%$ (272/844), respectively. Authors concluded topic modelling was a valuable sensitive method to detect non-compliance. However, it lacks specificity and manual review was required to distinguish between true and false positives in each dataset. They suggested that syntactic and semantic methods could be developed to recognize the experience, the temporal features, and the object concerned by the action in the sentences.

\section{Jones J, Pradhan M, Hosseini $M$, Kulanthaivel A, Hosseini M}

\section{Novel Approach to Cluster Patient- Generated Data Into Actionable Topics: Case Study of a Web-Based Breast Cancer Forum}

\section{JMIR Med Inform 2018;6(4):e45}

Despite the proliferation of social media use, such as blogs and forums, little is known about the scope and quality of information shared, or the purposes that social media sites serve for consumer decisional and support needs. This study explores approaches for analysing the free-text social media data to discover hidden, less obvious, aspects of health consumers' lives and extract potential valuable information on managing health and well being beyond the context of health care. This was applied to breast cancer management and recovery in five online breast cancer forums (mainly breastcancer.org community). Natural language processing and statistical modelling approach were used to cluster $>4$ million postings into manageable topics. Topic modelling (cluster of words that frequently occur together) was performed with the machine learning language toolkit open source tool. It was followed by multiple linear regression analysis to detect highly correlated topics among the different website forums. Quantitative content analysis of the forums resulted in 20 categories of user discussion. Topic model organized posts into 30 topics which were grouped into four distinct clusters of highly correlated computationally modelled topics. These clusters were labelled "symptoms and diagnosis", "treatment", "financial", "friends and family". Multiple regression analysis was performed to identify the most significant topics discussed among the forum participants. They were arranged in a descending order based on the Akaike information criterion value: 1) lingering side effects while in remission, 2) chemotherapy side effects and change of treatment, 3 ) radiation and side effects, 4) genetic risk and testing, 5) support from caregiver and medical team for long term recovery, and 6) looking for support from people in similar circumstances.

\section{Park A, Conway M, Chen AT}

Examining Thematic Similarity, Difference,
and Membership in Three Online Mental
Health Communities from Reddit: A Text
Mining and Visualization Approach

Comput Human Behav 2018 Jan; 78:98-112

Studies have consistently shown individuals can gain positive effects from interacting with other individuals in similar circumstances. Online interactions have been shown to improve depression, anxiety, stress, and negative mood, as well as to facilitate coping and empowerment. Moreover, members of online health communities consistently emphasize the benefits of participation with respect to their treatment decisions, symptom management, clinical management, and outcomes. In this study, authors examine the nature of online discussion (main themes expressed in the communities) and compare issues (thematic overlap, similarity and differences among the communities) pertaining to three mental health conditions: anxiety, depression, and post-traumatic stress disorder (PTSD). The corpus was based on Reddit (http://www.reddit.com), a popular social networking, online gathering, and news exchanging platform. Between the months of Oct 2015 to Dec 2015, a total of 7,410 posts and 132,599 associated comments made by 41,967 unique members were downloaded. Discussion themes were identified using knowledge resources like Unified Medical Language System or clusters analysis. Similarity among clusters in the network visualization used Louvain modularity algorithm. For each of the three main themes (anxiety, depression, and PTSD), 15 clusters had been generated. Using $\mathrm{r}$ /Anxiety subreddit discussion content, clusters including "social anxiety", "medication", "school", "panic attack", and "therapy/therapist" contained terms and labels which clearly differentiated the clusters from one another. A few clusters, such as "positive emotion" and "gratitude" shared terms. For the r/Depression subreddit, clusters including "birthday", "school", 
Lau et al.

"sleep", "work", and "gratitude" were clearly differentiated from one another. Clusters such as "talking to friends" and "friends and family" shared identical or semantically similar terms. For the r/PTSD subreddit, many clusters including "trauma therapy", "work", "sleep", "trauma trigger", "EMDR therapy", "nightmare", "animal", "research", were clearly distinguishable. A few clusters, such as "sleep" and "nightmare" shared similar terms but also had distinctive and non-overlapping terms. Venn diagrams were built to summarize and highlight common themes: "school" and "social related" between Anxiety Disorder and Depression, "living with" between Anxiety Disorder and PTSD. The global intersection between the three communities shared overlapping concerns and discussion patterns such as: "gratitude", "sleep", "work" and "positive emotion". However, Depression clusters focused on self-expressed concerns (e.g., events associated with depressed moods), whereas Anxiety Disorders and Post-Traumatic Stress Disorder clusters focused around treatmentand medication-related issues. 